PROCEEDINGS OF THE

AMERICAN MATHEMATICAL SOCIETY

Volume 133, Number 6, Pages 1741-1748

S 0002-9939(04)07844-X

Article electronically published on December 21, 2004

\title{
A SIMPLE CLOSURE CONDITION FOR THE NORMAL CONE INTERSECTION FORMULA
}

\author{
REGINA SANDRA BURACHIK AND VAITHILINGAM JEYAKUMAR
}

(Communicated by Jonathan M. Borwein)

\begin{abstract}
In this paper it is shown that if $C$ and $D$ are two closed convex subsets of a Banach space $X$ and $x \in C \cap D$, then $N_{C \cap D}(x)=N_{C}(x)+N_{D}(x)$ whenever the convex cone, (Epi $\sigma_{C}+$ Epi $\sigma_{D}$ ), is weak* closed, where $\sigma_{C}$ and $N_{C}$ are the support function and the normal cone of the set $C$ respectively. This closure condition is shown to be weaker than the standard interior-pointlike conditions and the bounded linear regularity condition.
\end{abstract}

\section{INTRODUCTION}

Never mind the regularity condition, in a normal cone intersection formula it's the interior-point-like condition [11, 15] which we need to avoid. The formula for closed convex sets is not always true without a regularity condition. The purpose of this paper is to show that a normal cone intersection formula for closed convex sets holds under a simple closure condition that is weaker than the interior-point-like conditions and bounded linear regularity condition [3, 4, 5].

A normal cone intersection formula states [7, 11, 4] that the normal cone of the intersection of sets equals the sum of the normal cones of the sets. A fundamental problem in convex analysis is to determine conditions under which the intersection formula holds at every point of the intersection of the sets. Such an intersection formula plays a key role in characterizing solutions of optimization problems and constrained best approximation problems. For instance, consider the optimization model problem

$$
\inf \{f(x) \mid x \in C \cap D\},
$$

where $C$ and $D$ are closed and convex subsets of a Banach $X$ and $f: X \rightarrow \mathbb{R} \cup\{\infty\}$ is a proper convex function. The model problem arises, for example, in convex

Received by the editors February 16, 2004.

2000 Mathematics Subject Classification. Primary 46N10, 90C25.

Key words and phrases. Normal cone, closure condition, bounded linear regularity, convex optimization, strong conical hull intersection property.

The first author's research was supported by CAPES (Grant BEX 0664-02/2), and was partially completed while the author was a visitor at the School of Mathematics, University of New South Wales, Sydney, and the School of Mathematics and Statistics, University of South Australia. This author wishes to thank the School of Mathematics at the University of South Australia for providing a stimulating environment and good infrastructure during her longer stay there, and also to the School of Mathematics at the University of New South Wales for their support. The authors are thankful to Jonathan Borwein for his helpful suggestions and for referring us to paper [4] and to the referee for the careful reading of the manuscript. 
programming problems [6, 14, 15], where $D=\left\{x \in X \mid g_{i}(x) \leq 0, i=1,2, \ldots, m\right\}$ and the $g_{i}$ 's are convex functions, and in constrained best approximation problems [9, 17], where $f(x)=\|y-x\|$ and $y \in X$. It is known that if $f$ is continuous at $x^{*} \in C \cap D$, then $x^{*}$ is an optimal solution of (1) if and only if

$$
0 \in \partial f\left(x^{*}\right)+N_{C \cap D}\left(x^{*}\right)
$$

where $\partial f$ is the subdifferential of $f$ and $N_{C \cap D}$ is the normal cone of the set $C \cap D$. The significance of this characterization relies on the description of $N_{C \cap D}\left(x^{*}\right)$ in terms of $N_{C}\left(x^{*}\right)$ and $N_{D}\left(x^{*}\right)$. If the interior-point condition that (int $\left.D\right) \cap C \neq \emptyset$ (or its recent generalization that the cone generated by $(C-D)$, cone $(C-D)$, is a closed subspace [13, 15, 10]) is satisfied or the bounded linear regularity condition [4, 5], when $X$ is a Euclidean space, is satisfied, then the normal cone intersection formula

$$
N_{C \cap D}(x)=N_{C}(x)+N_{D}(x), \forall x \in C \cap D,
$$

holds. The interior-point-like conditions are often restrictive in applications as for instance the set $D$ may not have interior points or the set $C$ may not have a point in the interior of $D$. For a simple example, let $C:=[0,1]$ and $D:=(-\infty, 0]$. Then $N_{C \cap D}(0)=\mathbb{R}=(-\infty, 0]+[0, \infty)=N_{C}(0)+N_{D}(0)$, and so the normal cone intersection formula holds at $x \in C \cap D=\{0\}$, whereas (int $D) \cap C=\emptyset$ and $\operatorname{cone}(C-D)=[0, \infty)$, which is not a subspace. For other examples, see [4, 5].

In this paper we show that if $C$ and $D$ are two closed convex subsets of a Banach space and $x \in C \cap D$, then $N_{C \cap D}(x)=N_{C}(x)+N_{D}(x)$ whenever the convex cone, (Epi $\sigma_{C}+$ Epi $\sigma_{D}$ ), is weak* closed, where $\sigma_{C}$ is the support function of the set $C$. We give a proof using a separation theorem [8, 12]. Our closure condition is shown to be weaker than the popularly known generalized interior point conditions and the bounded linear regularity condition [4, 5].

\section{Preliminaries}

We begin by fixing some definitions and notation. Let $X$ be a Banach space. The continuous dual space of $X$ will be denoted by $X^{\prime}$ and will be endowed with the weak* topology. For the set $D \subset \mathrm{X}$, the closure of $D$ and the interior of $D$ will be denoted $\operatorname{cl} D$ and int $D$ respectively. If a set $A \subset X^{\prime}, \operatorname{cl} A$ will stand for the weak* closure. Let $\operatorname{cone}(D)=\{\lambda x \mid \lambda \in \mathbb{R}, \lambda \geq 0, x \in D\}$. The indicator function $\delta_{D}$ is defined as $\delta_{D}(x)=0$ if $x \in D$ and $\delta_{D}(x)=+\infty$ if $x \notin D$. The support function $\sigma_{D}$ is defined by $\sigma_{D}(u)=\sup _{x \in D} u(x)$. The dual cone of $D$ is given by $D^{+}=\left\{\theta \in X^{\prime}: \theta(k) \geq 0, \forall k \in D\right\}$ and the normal cone of $D$ is given by $N_{D}(x):=\left\{v \in X^{\prime}: \sigma_{D}(v)=v(x)\right\}=\left\{v \in X^{\prime}: v(y-x) \leq 0, \forall y \in D\right\}$ when $x \in D$, and $N_{D}(x)=\emptyset$ when $x \notin D$.

Let $f: X \rightarrow \mathbb{R} \cup\{+\infty\}$ be a proper lower semi-continuous convex function. Then, the conjugate function of $f, f^{*}: X^{\prime} \rightarrow \mathbb{R} \cup\{+\infty\}$, is defined by

$$
f^{*}(v)=\sup \{v(x)-f(x) \mid x \in \operatorname{dom} f\}
$$

where the domain of $f, \operatorname{dom} f$, is given by

$$
\operatorname{dom} f=\{x \in X \mid f(x)<+\infty\} .
$$

The epigraph of $f$, Epi $f$, is defined by

$$
\text { Epi } f=\{(x, r) \in X \times \mathbb{R} \mid x \in \operatorname{dom} f, f(x) \leq r\} .
$$

Note that for a set $C \subset X, \delta_{C}^{*}=\sigma_{C}$. 
For the proper lower semi-continuous functions $f, g: X \rightarrow \mathbb{R} \cup\{+\infty\}$, the infimal convolution of $f$ with $g$ is denoted by $f \oplus g: X \rightarrow \mathbb{R} \cup\{+\infty\}$ and is defined by

$$
f \oplus g(x):=\inf _{x_{1}+x_{2}=x}\left\{f\left(x_{1}\right)+g\left(x_{2}\right)\right\} .
$$

The infimal convolution of $f$ with $g$ is said to be exact provided the infimum above is achieved for every $x \in X$. It is known (see, e.g., [19, Theorem 2.2(c)]) that if the infimal convolution is exact, then

$$
\text { Epi }(f \oplus g)=\text { Epi } f+\text { Epi } g \text {. }
$$

Moreover, if cone (dom $f$-dom $g$ ) is a closed subspace, then the infimal convolution of $f^{*}$ and $g^{*}$ is exact, and $f^{*} \oplus g^{*}=(f+g)^{*}$. For details, see [2, 19].

The conclusion of the following lemma, which plays a useful role in our development of a new closure condition, follows from a separation theorem.

Lemma 2.1. Let $C$ and $D$ be closed convex subsets of $X$. Then

$$
C \cap D \neq \emptyset \quad \Longleftrightarrow \quad(0,-1) \notin \operatorname{cl}\left(\text { Epi } \sigma_{C}+\text { Epi } \sigma_{D}\right) .
$$

Proof. (i) Let $(u, \alpha) \in\left(\right.$ Epi $\sigma_{C}+$ Epi $\left.\sigma_{D}\right)=$ Epi $\delta_{C}^{*}+$ Epi $\delta_{D}^{*}$. Then there exist $v, w \in X^{\prime}$, and $\beta, \delta \in \mathbb{R}$ such that $(v, \beta) \in$ Epi $\delta_{D}^{*},(w, \delta) \in$ Epi $\delta_{C}^{*}$, and $u=v+w$, $\alpha=\beta+\delta$. So, for each $x \in D, v(x) \leq \beta$, and for each $x \in C, w(x) \leq \delta$. Now, if $x \in A:=C \cap D$, then

$$
u(x)=(v+w)(x) \leq \beta+\delta=\alpha,
$$

which proves that $(u, \alpha) \in$ Epi $\sigma_{\mathrm{A}}$. This, together with the fact that Epi $\sigma_{A}$ is weak* closed, gives us

$$
\operatorname{cl}\left(\text { Epi } \delta_{D}^{*}+\text { Epi } \delta_{C}^{*}\right) \subset \text { epi } \sigma_{A} .
$$

If $C \cap D \neq \emptyset$, then clearly $(0,-1) \notin$ epi $\sigma_{A}$, and so from the above inclusion $(0,-1) \notin \operatorname{cl}\left(\right.$ Epi $\delta_{D}^{*}+$ Epi $\left.\delta_{C}^{*}\right)$. Conversely, if $(0,-1) \notin \operatorname{cl}\left(\right.$ Epi $\delta_{D}^{*}+$ Epi $\left.\delta_{C}^{*}\right)$, then by the separation theorem [[] $[\overline{]}$, Theorem 4.3.5] there is $(x, \alpha) \in X \times \mathbb{R},(x, \alpha) \neq$ $(0,0)$, such that $-\alpha<0$ and

$$
u(x)+\gamma \alpha \geq 0, \quad \forall(u, \gamma) \in \operatorname{cl}\left(\operatorname{Epi} \delta_{D}^{*}+\operatorname{Epi} \delta_{C}^{*}\right) .
$$

Let $\bar{x}=\frac{x}{\alpha}$. Then we have

$$
u(-\bar{x})-\gamma \leq 0, \forall(u, \gamma) \in \operatorname{cl}\left(\text { Epi } \delta_{D}^{*}+\text { Epi } \delta_{C}^{*}\right) .
$$

So, for each $v \in \operatorname{dom} \delta_{\mathrm{D}}^{*}$ and for each $w \in \operatorname{dom} \delta_{\mathrm{C}}^{*}$, we have $\left(v+w, \delta_{D}^{*}(v)+\delta_{C}^{*}(w)\right) \in$ $\operatorname{cl}\left(\right.$ Epi $\delta_{D}^{*}+$ Epi $\left.\delta_{C}^{*}\right)$ and hence,

$$
(v+w)(-\bar{x})-\delta_{D}^{*}(v)-\delta_{C}^{*}(w) \leq 0 .
$$

By letting $w=0$, we get $v(-\bar{x})-\delta_{D}^{*}(v) \leq 0$, for each $v \in \operatorname{dom} \delta_{D}^{*}$. Since $\delta_{D}$ is lower semi-continuous,

$$
\delta_{D}(-\bar{x})=\delta_{D}^{* *}(-\bar{x})=\sup _{v}\left[v(-\bar{x})-\delta_{D}^{*}(v)\right] \leq 0 .
$$

This implies that $-\bar{x} \in D$. Similarly, we can show that $-\bar{x} \in C$. Thus $-\bar{x} \in$ $C \cap D \neq \emptyset$. 


\section{ThE NORMAL CONE INTERSECTION FORMULA}

In this section we derive the normal cone intersection formula for closed convex sets. We first obtain a key extension of the dual cone intersection formula for closed convex cones $C$ and $D$ such that $(C \cap D)^{+}=\operatorname{cl}\left(C^{+}+D^{+}\right)$to closed convex sets $C$ and $D$ which are not necessarily cones. The extension, which is expressed in terms of the epigraphs of the support functions of $C$ and $D$, then leads to a closure condition, ensuring the normal cone intersection formula.

Lemma 3.1. Let $C$ and $D$ be closed convex subsets of $X$. If $C \cap D \neq \emptyset$, then

$$
\text { Epi } \sigma_{C \cap D}=\operatorname{cl}\left(\text { Epi } \sigma_{C}+\text { Epi } \sigma_{D}\right) \text {. }
$$

Proof. Let $A:=C \cap D \neq \emptyset$. Then, as we saw in the proof of Lemma 2.1 (see (44)), we have the inclusion

$$
\operatorname{cl}\left(\operatorname{Epi} \sigma_{D}+\text { Epi } \sigma_{C}\right) \subset \text { Epi } \sigma_{A} .
$$

To show the reverse inclusion, let $(u, \alpha) \notin \operatorname{cl}\left(\operatorname{Epi} \sigma_{D}+\operatorname{Epi} \sigma_{C}\right)$. Since $A \neq \emptyset$, it follows from Lemma 2.1 that $(0,-1) \notin \mathrm{cl}\left(\right.$ Epi $\left.\sigma_{D}+\operatorname{Epi} \sigma_{C}\right)$. So,

$$
B \cap\left(\operatorname{cl}\left(\operatorname{Epi} \sigma_{D}+\operatorname{Epi} \sigma_{C}\right)\right)=\emptyset,
$$

where $B:=\left\{\delta(u, \alpha)+(1-\delta)(0,-1) \in X^{\prime} \times \mathbb{R} \mid \delta \in[0,1]\right\}$ is the segment connecting the points $(u, \alpha)$ and $(0,-1)$. Otherwise, there is $\delta_{0} \in(0,1)$ such that

$$
\delta_{0}(u, \alpha)+\left(1-\delta_{0}\right)(0,-1) \in \operatorname{cl}\left(\operatorname{Epi} \sigma_{D}+\operatorname{Epi} \sigma_{C}\right) ;
$$

thus,

$$
\left(\delta_{0} u, \delta_{0} \alpha-\left(1-\delta_{0}\right)\right) \in \operatorname{cl}\left(\operatorname{Epi} \sigma_{D}+\operatorname{Epi} \sigma_{C}\right) .
$$

Also $\{0\} \times \mathbb{R}_{+} \subset \operatorname{cl}\left(\operatorname{Epi} \sigma_{D}+\operatorname{Epi} \sigma_{C}\right)$. Then,

$$
\left(\delta_{0} u, \delta_{0} \alpha\right)=\left(\delta_{0} u, \delta_{0} \alpha-\left(1-\delta_{0}\right)\right)+\left(0,1-\delta_{0}\right) \in \operatorname{cl}\left(\text { Epi } \sigma_{D}+\text { Epi } \sigma_{C}\right) .
$$

This gives us that

$$
(u, \alpha)=\frac{1}{\delta_{0}}\left(\delta_{0} u, \delta_{0} \alpha\right) \in \operatorname{cl}\left(\operatorname{Epi} \sigma_{D}+\operatorname{Epi} \sigma_{C}\right),
$$

which is a contradiction.

Now, by applying the separation theorem, there is $(x, \beta) \in X \times \mathbb{R},(x, \beta) \neq(0,0)$ such that

and

$$
[\delta(u, \alpha)+(1-\delta)(0,-1)](x, \beta)<0, \quad \forall \delta \in[0,1]
$$

$$
v(x)+\gamma \beta \geq 0, \forall(v, \gamma) \in \operatorname{cl}\left(\operatorname{Epi} \sigma_{D}+\operatorname{Epi} \sigma_{C}\right) .
$$

By letting $\delta=0$ we get $\beta>0$ and by letting $\delta=1$ we obtain $u(x)+\alpha \beta<0$; thus, $u\left(\frac{-x}{\beta}\right)>\alpha$. Also, the same argument as in the proof of Lemma 2.1 leads to

$$
\frac{-x}{\beta} \in C \cap D .
$$

This together with the fact that $u\left(\frac{-x}{\beta}\right)>\alpha$ implies that $(u, \alpha) \notin \operatorname{Epi} \sigma_{A}$.

Observe that if $C$ and $D$ are closed and convex cones of $X$, then the conclusion of Lemma 3.1 gives us that

$-(C \cap D)^{+} \times \mathbb{R}_{+}=\operatorname{Epi} \sigma_{C \cap D}=\operatorname{cl}\left(\right.$ Epi $\left.\sigma_{C}+\operatorname{Epi} \sigma_{D}\right)=\operatorname{cl}\left[\left(-C^{+}-D^{+}\right) \times \mathbb{R}_{+}\right]$, and so, $(C \cap D)^{+}=\operatorname{cl}\left(C^{+}+D^{+}\right)$. 
We now derive the main result as an application of Lemma 3.1. Note that the set (Epi $\sigma_{C}+$ Epi $\left.\sigma_{D}\right)$ is a convex cone.

Theorem 3.1. Let $X$ be a Banach space and let $C$ and $D$ be two closed and convex subsets of $X$. If the set (Epi $\sigma_{C}+$ Epi $\left.\sigma_{D}\right)$ is weak* closed, then

$$
\forall x \in C \cap D, \quad N_{C \cap D}(x)=N_{C}(x)+N_{D}(x) .
$$

Proof. Let $x \in C \cap D$. Then, clearly $N_{C \cap D}(x) \supset N_{C}(x)+N_{D}(x)$. To show the reverse inclusion, let $v \in N_{C \cap D}(x)$. This means that $\sigma_{C \cap D}(v)=v(x)$, which in turn gives $(v, v(x)) \in$ Epi $\sigma_{C \cap D}$. Since Epi $\sigma_{C}+$ Epi $\sigma_{D}$ is weak* closed, it follows from Lemma 3.1 that $(v, v(x)) \in$ Epi $\sigma_{C}+$ Epi $\sigma_{D}$. Then there exist two elements $\left(v_{1}, \alpha_{1}\right) \in$ Epi $\sigma_{C}$ and $\left(v_{2}, \alpha_{2}\right) \in$ Epi $\sigma_{D}$ such that $v_{1}+v_{2}=v$ and $\alpha_{1}+\alpha_{2}=v(x)$. So,

$$
v(x)=\alpha_{1}+\alpha_{2} \geq \sigma_{C}\left(v_{1}\right)+\sigma_{D}\left(v_{2}\right) \geq v_{1}(x)+v_{2}(x)=v(x),
$$

which gives us $\sigma_{C}\left(v_{1}\right)+\sigma_{D}\left(v_{2}\right)=v(x)$. Now,

$$
\begin{aligned}
0 \geq v_{1}(x)-\sigma_{C}\left(v_{1}\right) & =\left(v-v_{2}\right)(x)+\sigma_{D}\left(v_{2}\right)-v(x)=\sigma_{D}\left(v_{2}\right)-v_{2}(x) \\
& \geq v_{2}(z)-v_{2}(x)=v_{2}(z-x),
\end{aligned}
$$

for each $z \in D$. Thus, $v_{2} \in N_{D}(x)$. Similarly, we can show also that $v_{1} \in N_{C}(x)$. Hence, $v \in N_{C}(x)+N_{D}(x)$.

We now see that Theorem 3.1 leads to the sum formula for dual cones under our closure condition. Note that if $0 \in C$, it follows from the definitions that $N_{C}(0)=-C^{+}$.

Corollary 3.1. Let $C$ and $D$ be two closed and convex subsets of $X$ such that $0 \in C \cap D$ and the set (Epi $\sigma_{C}+$ Epi $\left.\sigma_{D}\right)$ is weak ${ }^{*}$ closed. Then

$$
(C \cap D)^{+}=C^{+}+D^{+} \text {. }
$$

Proof. Clearly, $N_{C}(0)=-C^{+}$and $N_{D}(0)=-D^{+}$. It follows from Theorem 3.1 that $N_{C \cap D}(0)=N_{C}(0)+N_{D}(0)$ and hence

$$
-(C \cap D)^{+}=-\left(C^{+}\right)-\left(D^{+}\right)=-\left(C^{+}+D^{+}\right),
$$

which readily implies (5).

We also see that the known interior-point-like conditions yield our closure condition. Recall that $\operatorname{core}(A):=\{a \in A \mid(\forall x \in X)(\exists \varepsilon>0)$ such that $(\forall \lambda \in$ $[-\varepsilon, \varepsilon]) a+\lambda x \in A\}$.

Proposition 3.1. Let $C$ and $D$ be closed and convex subsets of $X$. Then the set (Epi $\sigma_{C}+$ Epi $\left.\sigma_{D}\right)$ is weak ${ }^{*}$ closed if one of the following conditions holds:

(i) (int $D) \cap C \neq \emptyset$,

(ii) $0 \in \operatorname{core}(C-D)$,

(iii) $\operatorname{cone}(C-D)$ is a closed subspace.

Proof. Clearly, (i) implies (ii), which in turn implies (iii). So, it suffices if we show that (iii) implies that the set (Epi $\sigma_{C}+$ Epi $\sigma_{D}$ ) is weak*-closed. Indeed, if (iii) holds, then, using [19] Theorem 3.6], we get $\sigma_{D \cap C}=\sigma_{D} \oplus \sigma_{C}$, with exact infimal convolution. As a consequence of the exactness (see equation (3)), we have that Epi $\sigma_{C \cap D}=$ Epi $\sigma_{C}+$ Epi $\sigma_{D}$. Since the set in the left-hand side is weak* closed, the conclusion holds. 
The following simple example illustrates the situation where the conditions (i)(iii) fail; whereas our closure condition holds.

Example 3.1. Let $C:=[0,1]$ and $D:=(-\infty, 0]$. Then $N_{C \cap D}(0)=\mathbb{R}=(-\infty, 0]+$ $[0, \infty)=N_{C}(0)+N_{D}(0)$, and so the normal cone intersection formula holds at $x \in C \cap D=\{0\}$, whereas $(\operatorname{int} D) \cap C=\emptyset$ and cone $(C-D)=[0, \infty)$, which is not a subspace. However, Epi $\sigma_{C}+\operatorname{Epi} \sigma_{D}=\{(x, t) \in \mathbb{R} \times \mathbb{R} \mid \max \{x, 0\} \leq t\}+\mathbb{R}_{+} \times \mathbb{R}_{+}=$ $\mathbb{R} \times \mathbb{R}_{+}$is a closed convex cone.

For related conditions guaranteeing the closure of the sum of two closed convex sets, see [1].

\section{Regularity AND ClOSED CONVEX CONES}

We now examine the connections between our closure condition and the bounded linear regularity condition 4, 5, in the case where $C$ and $D$ are closed and convex cones.

Proposition 4.1. Let $C$ and $D$ be closed convex cones of $X$. Then the set (Epi $\sigma_{C}+$ Epi $\left.\sigma_{D}\right)$ is weak* closed if and only if $\left(C^{+}+D^{+}\right)$is weak $k^{*}$ closed.

Proof. If the set (Epi $\sigma_{C}+$ Epi $\sigma_{D}$ ) is weak* closed, then it follows from Corollary 3.1 that $\left(C^{+}+D^{+}\right)=(C \cap D)^{+}$, which is weak* closed. Conversely, assume that $C^{+}+D^{+}$is weak ${ }^{*}$ closed. Since $C$ and $D$ are closed convex cones,

$$
-\left(C^{+}+D^{+}\right) \times \mathbb{R}_{+}=\left(-C^{+} \times \mathbb{R}_{+}\right)+\left(-D^{+} \times \mathbb{R}_{+}\right)=\text {Epi } \sigma_{C}+\text { Epi } \sigma_{D} .
$$

Hence, if $\left(C^{+}+D^{+}\right)$is weak* closed, then the set (Epi $\sigma_{C}+$ Epi $\left.\sigma_{D}\right)$ is weak* closed.

In the case where $X$ is a Euclidean space, we will now show that our closure condition is equivalent to the normal cone intersection formula for closed convex cones. Recall that the negative dual cone of a set $D$ is given by $D^{-}:=-D^{+}$.

Proposition 4.2. Let $X$ be a Euclidean space. Let $C$ and $D$ be closed convex cones of $X$. Then the following statements are equivalent.

(i) The set (Epi $\sigma_{C}+$ Epi $\left.\sigma_{D}\right)$ is closed.

(ii) $\left(C^{+}+D^{+}\right)$is closed.

(iii) For each $x \in C \cap D, N_{C \cap D}(x)=N_{C}(x)+N_{D}(x)$.

Proof. [(i) $\Longleftrightarrow$ (ii)]. This follows from Proposition 4.1 .

[(ii) $\Longleftrightarrow$ (iii)]. The set $C^{+}+D^{+}$is closed if and only if $C^{-}+D^{-}$is closed. Now, the equivalence follows from Proposition 4.1 and Proposition 20 of [4].

Recall that the pair $\{C, D\}$ is said to be boundedly linearly regular [3] if for every bounded set $S$ in $X$, there exists $\kappa_{S}>0$ such that the distances to the sets $C, D$ and $C \cap D$ are related by

$$
d(x, C \cap D) \leq \kappa_{S} \max \{d(x, C), d(x, D)\},
$$

for every $x \in S$, where $d(x, C):=\inf \{\|x-c\| \mid c \in C\}$ is the distance function.

Proposition 4.3. Let $X$ be a Euclidean space. Let $C$ and $D$ be closed convex cones of $X$. If the pair $\{C, D\}$ is boundedly linearly regular, then the set (Epi $\sigma_{C}+$ Epi $\sigma_{D}$ ) is closed. 
Proof. Theorem 3 of [4] gives us that bounded linear regularity implies that the normal cone intersection formula, called strong CHIP in [4, holds for closed convex sets. Hence, the conclusion follows from Proposition 4.2.

Note that in the case where $C$ and $D$ are closed convex cones in a Euclidean space, the condition that the set (Epi $\sigma_{C}+$ Epi $\sigma_{D}$ ) is closed does not imply that the pair $\{C, D\}$ is boundedly linearly regular. Indeed, it has recently been shown in [5. Corollary 3.2] that the normal cone intersection formula may hold for certain closed convex cones $C$ and $D$, whereas the pair $\{C, D\}$ is not boundedly linearly regular. Thus, the counterexample in $\mathbb{R}^{4}$ in Section 3 of [5] shows that the set (Epi $\sigma_{C}+$ Epi $\left.\sigma_{D}\right)$ is closed, whereas the pair $\{C, D\}$ is not boundedly linearly regular.

On the other hand, Proposition 5.16 of $[3$ shows that if $C$ and $D$ are closed subspaces in a Hilbert space, then $\left(C^{\perp}+D^{\perp}\right)$ is closed if and only if $\{C, D\}$ is boundedly linearly regular. Note in this case that $\left(C^{\perp}+D^{\perp}\right)$ is closed if and only if the set Epi $\sigma_{C}+\operatorname{Epi} \sigma_{D}$ is closed. This follows from the fact that Epi $\sigma_{C}+\operatorname{Epi} \sigma_{D}=$ $\left(C^{\perp} \times \mathbb{R}_{+}\right)+\left(D^{\perp} \times \mathbb{R}_{+}\right)=\left(C^{\perp}+D^{\perp}\right) \times \mathbb{R}_{+}$, where the set $V^{\perp}$ is the subspace perpendicular to the subspace $V$.

Hence, if $C$ and $D$ are closed subspaces in a Hilbert space, then the following conditions are equivalent:

(i) The set (Epi $\sigma_{C}+$ Epi $\left.\sigma_{D}\right)$ is closed.

(ii) $\left(C^{\perp}+D^{\perp}\right)$ is closed.

(iii) $\{C, D\}$ is boundedly linearly regular.

We end this section by pointing out that, in the particular case in which $C$ and $D$ are subspaces of a Euclidean space $X$, the set Epi $\sigma_{C}+$ Epi $\sigma_{D}$ is always closed, since the subspace $\left(C^{\perp}+D^{\perp}\right)$ is always closed in a Euclidean space.

\section{Conclusion And Further Research}

In this paper we have shown that the normal cone intersection formula for closed convex sets holds under a simple closure condition. In other words, we have established the subdifferential sum formula [2, 4, 16, that $\partial\left(\delta_{C}+\delta_{D}\right)(x)=$ $\partial \delta_{C}(x)+\partial \delta_{D}(x)$, for the indicator functions of two closed convex sets $C$ and $D$ under a closure condition that is much weaker than the interior-point-like conditions. The following questions naturally arise: Does the subdifferential sum formula for two arbitrary proper lower semi-continuous convex functions hold under a similar closure condition that is weaker than the interior-point-like conditions? Is the pointwise sum of two maximal monotone operators a maximal monotone operator under an appropriate extension of our closure condition? The answers to these questions appear to be in the affirmative and will be investigated in a further study.

\section{REFERENCES}

[1] S. Adly, E. Emil and M. Thera, On the closedness of the algebraic difference of closed convex sets, J. Math. Pures Appl., 82(9) (2003), 1219-1249. MR2012809 (2004g:49028)

[2] H. Attouch and H. Brezis, Duality for the sum of convex functions in general Banach spaces, in Aspects of Mathematics and Its Applications, J. A. Barroso, ed., Elsevier, Amsterdam, 1986, 125-133. MF 0849549 (87m:90095)

[3] H. H. Bauschke and J. M. Borwein, On projection algorithms for solving convex feasibility problems, SIAM. Rev., 38 (1993), 367-426. MR1409591 (98f:90045) 
[4] H. H. Bauschke, J. M. Borwein and W. Li, Strong conical hull intersection property, bounded linear regularity, Jameson's property $(\mathrm{G})$, and error bounds in convex optimization, Math. Progr., 86 (1999), 135-160. MR.1712477|(2000f:90095)

[5] H. H. Bauschke, J. M. Borwein and P. Tseng, Bounded linear regularity, strong CHIP, and CHIP are distinct properties, J. Convex Analysis, 7(2) (2000), 395-412. MR1811687 (2002d:90113)

[6] J. M. Borwein and H. Wolkowicz, A simple constraint qualification in infinite dimensional programming, Math. Progr., 35 (1986), 83 - 96. MR0842636 (87i:90321)

[7] M. Ciligot-Travain, An intersection formula for the normal cone associated with the hypertangent cone, Appl. Anal., 5 (1999), 239-247. MR.1722221 (2000h:49025)

[8] M. Cotlar and R. Cignoli, An Introduction to Functional Analysis, North-Holland Publishing Company, The Netherlands, 1974. MR0405049 (53:8845)

[9] F. Deutsch, W. Li and J. D. Ward, Best approximation from the intersection of a closed convex set and a polyhedron in Hilbert space, weak Slater conditions, and the strong conical hull intersection property, SIAM J. Optim., 10 (1999), 252-268. MR1742319 (2001a:41047)

[10] M.S. Gowda and M. Teboulle, A comparison of constraint qualifications in infinitedimensional convex programming, SIAM J. Control and Optim., 28 (4), 1990, 925 - 935. MR 1051630 (91f:90100)

[11] J. B. Hiriart-Urruty and C. Lemarechal, Convex Analysis and Minimization Algorithms, Volumes I and II, Springer-Verlag, Berlin Heidleberg, 1993. MF 1261420 (95m:90001) MR 1295240 (95m:90002)

[12] R. B. Holmes, Geometric Functional Analysis and its Applications, Springer-Verlag, Berlin, 1975. MR0410335 (53:14085)

[13] V. Jeyakumar, Duality in infinite dimensional optimization, Nonlinear Anal. T,ME A, 15 (1990), 1111-1122. MF $1082286(91 \mathrm{k}: 49053)$

[14] V. Jeyakumar, G. M. Lee and N. Dinh, New sequential Lagrange multiplier conditions characterizing optimality without constraint qualification for convex programs, SIAM J. Optim., 14(2) (2003), 534-547. MR2048156

[15] V. Jeyakumar and H. Wolkowicz, Generalizations of Slater's constraint qualification for infinite convex programs, Math. Progr., 57(1) (1992), 85-102. MR1167408 (93e:90070)

[16] F. Jules and M. Lassonde, Formulas for subdifferentials of sums of convex functions, J. Convex Anal., 9(2), (2002), 519-533. MR1970570 (2004b:49029)

[17] C. Li and X. Jin, Nonlinearly constrained best approximation in Hilbert spaces: the strong CHIP, and the basic constraint qualification, SIAM J. Optim., 13(1) (2002), 228-239. MR 1922763 (2003k:41048)

[18] T. Rockafellar, Convex Analysis, Princeton University Press, Princeton, (1970), NJ. MR $0274683(43: 445)$

[19] T. Strömberg, The operation of infimal convolution, Diss. Math., 352, (1996), 1-61. MR.1387951 (97c:49018)

Engenharia de Sistemas e Computacao, COPPE-UFRJ CP 68511, Rio de Janeiro-RJ, CEP 21945-970, BRAZIL

E-mail address: regi@cos.ufrj.br

Department of Applied Mathematics, University of New South Wales, Sydney 2052, Australia

E-mail address: jeya@maths.unsw.edu.au 